\title{
New infra-red enhancements in 4-derivative gravity
}

\author{
Alberto Salvio $^{1, \mathrm{a}}$, Alessandro Strumia ${ }^{1,2}$, Hardi Veermäe ${ }^{1,3}$ \\ ${ }^{1}$ Theoretical Physics Department, CERN, Geneva, Switzerland \\ 2 Dipartimento di Fisica dell'Università di Pisa and INFN, Pisa, Italy \\ ${ }^{3}$ National Institute of Chemical Physics and Biophysics, Tallinn, Estonia
}

Received: 3 September 2018 / Accepted: 4 October 2018 / Published online: 20 October 2018

(C) The Author(s) 2018

\begin{abstract}
Derivative gravity provides a renormalizable theory of quantum gravity at the price of introducing a physical ghost, which could admit a sensible positive-energy quantization. To understand its physics, we compute ghostmediated scatterings among matter particles at tree-level, finding a new power-like infra-red enhancement typical of 4-derivative theories, that we dub 'ghostrahlung'. SuperPlanckian scatterings get downgraded to Planckian by radiating hard gravitons and ghosts, which are weakly coupled and carry away the energy.
\end{abstract}

\section{Contents}

1 Introduction . . . . . . . . . . . . . . . 1

2 Rates for gravi-ghost-mediated processes ..... . 2

3 Specific processes ............ 5

4 Ghostrahlung .............. . 7

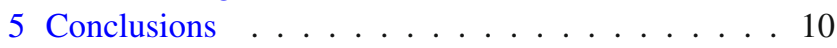

References ................ 11

\section{Introduction}

4-Derivative gravity provides a renormalizable gravity theory [1], controlled by dimensionless coupling constants, called $f_{2}$ and $f_{0}$ in [2]. In the presence of the dimension-full superrenormalizable Einstein term, the graviton splits into $g$ (the massless graviton) and $g_{2}$ (a spin-2 state with mass $M_{2}$; an extra spin 0 component $g_{0}$ with mass $M_{0}$ is less relevant) as clear from the decomposition of its propagator, with Lorentz indices omitted

$$
P\left(k^{2}\right)=\frac{1}{M_{2}^{2} k^{2}-k^{4}}=\frac{1}{M_{2}^{2}}\left[\frac{1}{k^{2}}-\frac{1}{k^{2}-M_{2}^{2}}\right] .
$$

a e-mail: alberto.salvio@roma2.infn.it
This cancellation between $g$ and $g_{2}$ in the virtual propagator makes gravity renormalizable; however, the minus sign means that $g_{2}$ classically has negative kinetic energy. The same problem was encountered with classical fermions. 4derivative theories too admit a positive-energy quantization, but at the price of an indefinite quantum norm that obscures the probabilistic interpretation [3-7]. In view of this situation, we here explore how the ghost behaves making two pragmatic simplifications.

1. First, we restrict the attention to observables measurable from asymptotic distance: life-times and cross sections. A ghost is then indirectly defined through its effects as an intermediate virtual particle in Feynman diagrams that describe scatterings among matter particles (scalars, fermions and vectors).

This is how collider experiments reconstruct any short-lived particle from kinematical distributions of final-state particles. When the intermediate particle is a ghost, this is known as 'Lee-Wick approach' [8-11], and ambiguities appear at higher orders, in diagrams that probe configurations with two ghosts.

2. Second, we focus on tree-level processes, not affected by higher order ambiguities, and that can probe the generic gravi-ghost kinematics. Figure 1 shows an example of this.

Under these assumptions, we will extract a good deal of ghost physics, common to various attempts of fully defining the theory. The theory is well defined in the Euclidean space: according to [12] a generalization of the Wick rotation defines Minkowskian physics solving the above-mentioned ambiguities perturbatively to all orders. When evaluating tree-level diagrams their approach reduces to ours: integrated cross sections are not affected by the extra structure assumed in 

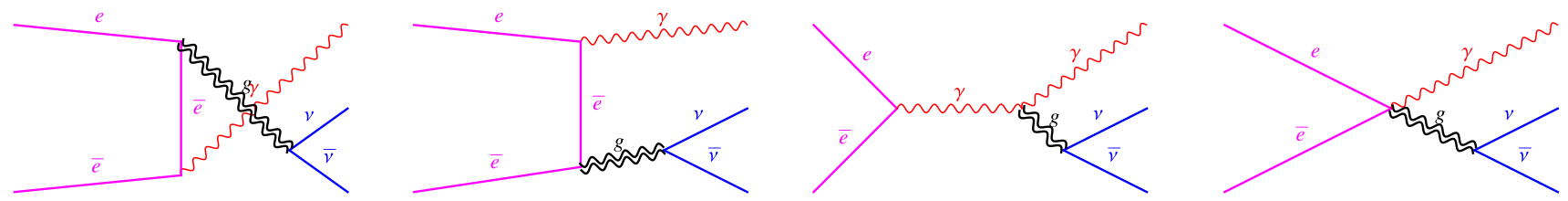

Fig. 1 Sample of gravi-ghost-mediated $2 \rightarrow 3$ process: the differential distribution of $\sigma(e \bar{e} \rightarrow \gamma \nu \bar{v})$ is used to infer the cross section for $\sigma(e \bar{e} \rightarrow \gamma g)$

[12] on the top of the ghost resonance; all momenta that enter our expressions are Minkowskian physical observables described by real numbers. The other approaches that extract probabilities from negative norms $[4,6,7]$ similarly reduce to our results when evaluated at leading order in the couplings.

Cross sections in QED and QCD are affected by soft and collinear infra-red (IR) divergences. These effects are well understood thanks to soft theorems [13-15], which also apply to Einstein gravity [16-18]. We find a new kind of IR enhancement related to the 4-derivative structure and to the consequent gravi-ghost propagator of Eq. (1). Cross sections mediated by a gravi-ghost contain a factor $f_{2}^{2} s \int d\left(k^{2}\right) P\left(k^{2}\right)$. In the limit of massless gravi-ghost this factor is power IR divergent at small $k^{2}$. As a result, cross sections such as $e^{+} e^{-} \rightarrow \gamma \nu \bar{v}$ (Fig. 1) do not have the form expected in theories with dimensionless coupling, $\sigma \sim 1 / s$ times powers of the couplings. Indeed, in the massless limit, the Newton potential $V \propto 1 / r$ gets replaced by a confining $V \propto r$ : free particles disappear in this limit.

In the realistic massive theory, the IR divergence is cut by the gravi-ghost mass $M_{2}$. Then each massive gravi-ghost contributes to the cross section with a multiplicative enhancement $f_{2}^{2} s / M_{2}^{2} \sim s / M_{\mathrm{Pl}}^{2}$. Gravi-ghost radiation becomes an order one correction in super-Planckian collisions. While purely gravitational cross sections can remain smaller than in Einstein theory, cross sections mediated by large matter couplings (such as Fig. 1) look as bad as those in UV-divergent Einstein theory, which violate naive perturbative unitarity. This reassures that ghosts do not do miracles, like cancelling positive cross sections with negative cross sections.

In agravity cross sections grow because of the new IR enhancement of gravi-ghost emission. Unlike in Einstein gravity, Planckian gravitons negligibly interact and simply carry away their energy. As a result energies above the Planck scale get radiated down to sub-Planckian, energies without forming, at the same time, non-perturbative structures such as black holes.

An additional issue is that the IR enhancement is saturated at the ghost pole. To understand what it is, one needs to go beyond perturbation theory. As well known, perturbative corrections diverge when an intermediate particle goes onshell. A non-perturbative resummation transforms a matter pole into a Breit-Wigner peak, $1 /\left(k^{2}-m^{2}+i k \Gamma\right)$.
A matter particle acquires a decay width $\Gamma$ with the same sign as the $i \epsilon$ prescription in its Feynman propagator, $1 /\left(k^{2}-\right.$ $\left.m^{2}+i \epsilon\right)$, which defines the theory as the continuation from the Euclidean known as 'Wick rotation'. On the other hand, a massive ghost that decays into matter particles acquires a negative decay width $\Gamma<0$, which makes its behaviour acausal on microscopic scales [10,11].

The paper is structured as follows. In Sect. 2 we derive rates of generic gravi-ghost-mediated processes. In Sect. 3 we compute specific examples. In Sect. 4 we interpret IRenhanced rates. Conclusions are given in Sect. 5.

\section{Rates for gravi-ghost-mediated processes}

\subsection{Summary of the theory and of notations}

Following [1] we consider the renormalizable action (in the notation of [2])

$S=\int d^{4} x \sqrt{|\operatorname{det} g|}\left[\frac{R^{2}}{6 f_{0}^{2}}+\frac{\frac{1}{3} R^{2}-R_{\mu \nu}^{2}}{f_{2}^{2}}-\frac{\bar{M}_{\mathrm{Pl}}^{2}}{2} R+\mathscr{L}_{\text {matter }}\right]$,

where the first two terms, suppressed by the dimensionless gravitational couplings $f_{0}$ and $f_{2}$, are the 4-derivative graviton kinetic terms; the latter term $\mathscr{L}_{\text {matter }}$ is the part of the Lagrangian that depends on the matter fields (scalars $S$, fermions $\psi$, vectors $V$ with gauge-covariant kinetic terms, Yukawa couplings, quartic scalars, scalar couplings to gravity, $-\xi_{S}|S|^{2} R$ and possibly with super-renormalizable terms).

The Einstein-Hilbert term, in the middle, could be induced dynamically from a dimensionless action, e.g. as $\bar{M}_{\mathrm{Pl}}^{2} / 2=$ $\xi_{S}\langle S\rangle^{2}$ [2]. In its presence, the 4-derivative graviton splits into the massless spin-2 graviton, a spin-2 ghost $g_{2}$ with mass $M_{2}=f_{2} \bar{M}_{\mathrm{Pl}} / \sqrt{2}$, a spin-0 scalar $g_{0}$ with mass $M_{0}=$ $f_{0} \bar{M}_{\mathrm{Pl}} / \sqrt{2}$. We collectively denote them as 'gravi-ghost'. The gravi-ghost propagator is (in the gauge $\xi_{h}=c_{g}=0$ of [2])

$P_{\mu \nu \alpha \beta}\left(k^{2}\right)=i \sum_{j=\{0,2\}} c_{j} f_{j}^{2} P^{(j)}\left(k^{2}\right) \Pi_{\mu \nu \alpha \beta}^{(j)}$, 


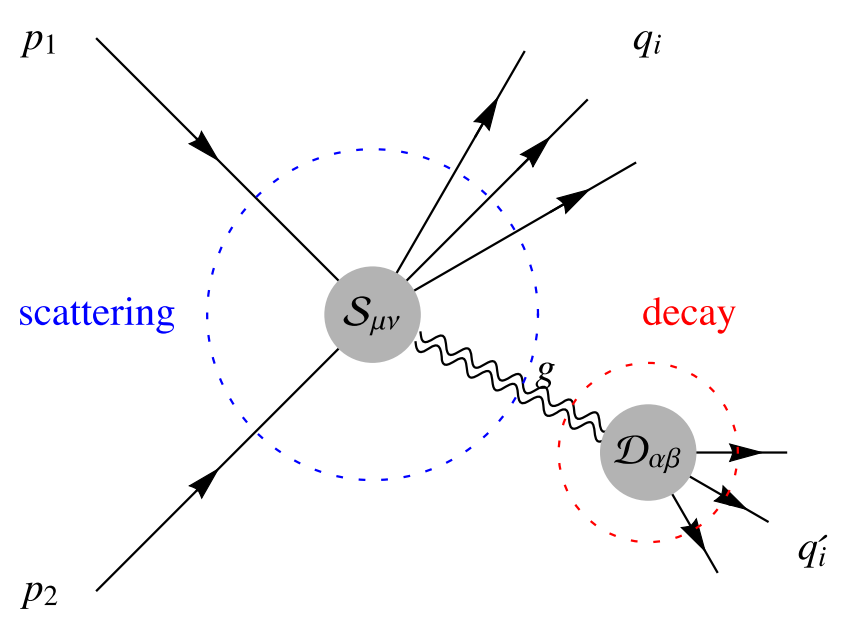

Fig. 2 A generic process among matter particles mediated by one gravi-ghost, denoted as a double wave

where $k_{\mu}$ is the quadri-momentum, $c_{0}=1, c_{2}=-2$ (this sign is crucial) and

$P^{(2)}\left(k^{2}\right)=\frac{1}{k^{2}\left(k^{2}-M_{2}^{2}+i k \Gamma_{2}\right)}$,

$P^{(0)}\left(k^{2}\right)=\frac{1}{k^{2}\left(k^{2}-M_{0}^{2}+i k \Gamma_{0}\right)}$.

We added decay widths $\Gamma_{j}$ which appear after (naively) resumming quantum corrections to the propagator. The $\Pi_{\mu \nu \alpha \beta}^{(j)}$ are orthogonal projectors over the spin components $[1,2]$, which sum up to unity: $\left(\Pi^{(2)}+\Pi^{(1)}+\Pi^{(0)}+\right.$ $\left.\Pi^{(0 w)}\right)_{\mu \nu \alpha \beta}=\frac{1}{2}\left(\eta_{\mu \nu} \eta_{\alpha \beta}+\eta_{\mu \beta} \eta_{\alpha \nu}\right)$. We only need $\Pi^{(2)}$ and $\Pi^{(0)}$, which are

$\Pi_{\mu \nu \rho \sigma}^{(2)}=\frac{1}{2} T_{\mu \rho} T_{\nu \sigma}+\frac{1}{2} T_{\mu \sigma} T_{\nu \rho}-\frac{T_{\mu \nu} T_{\rho \sigma}}{3}$,

$\Pi_{\mu \nu \rho \sigma}^{(0)}=\frac{T_{\mu \nu} T_{\rho \sigma}}{3}$,

where $T_{\mu \nu}=\eta_{\mu \nu}-k_{\mu} k_{\nu} / k^{2}$ is the transverse projector.

\subsection{Processes mediated by one gravi-ghost}

In order to study the gravi-ghost behaviour we consider a generic scattering between matter particles that contains one intermediate virtual gravi-ghost $g$ with quadri-momentum $k_{\mu}$ that transforms into matter particles with quadri-momenta $q_{i}^{\prime}$, as illustrated in Fig. 2. We denote the quadri-momenta of the other final-state particles as $q_{i}$, and those of the two initialstate particles as $p_{1}, p_{2}$, such that momentum conservations reads $\sum p_{i}=\sum q_{i}+\sum q_{i}^{\prime}$. We decompose the process as a 'scattering' with momenta $\sum p_{i}=k+\sum q_{i}$ times a gravighost 'decay' with momenta $k=\sum q_{i}^{\prime}$. Concrete examples are $2 \rightarrow 2$ scatterings such as $e\left(p_{1}\right) \bar{e}\left(p_{2}\right) \rightarrow g(k) \rightarrow$ $v\left(q_{1}^{\prime}\right) \bar{v}\left(q_{2}^{\prime}\right)$ (where $k^{2}$ is fully determined by kinematics) and
$2 \rightarrow 3$ scatterings such as $e\left(p_{1}\right) \bar{e}\left(p_{2}\right) \rightarrow \gamma\left(q_{1}\right) v\left(q_{1}^{\prime}\right) \bar{v}\left(q_{2}^{\prime}\right)$ as shown in Fig. 1.

The cross section is $d \sigma / d \Phi=|\mathscr{A}|^{2} / 4 I$, where $I \equiv$ $\sqrt{\left(p_{1} \cdot p_{2}\right)^{2}-m_{1}^{2} m_{2}^{2}}$ is the usual flux factor $(I=s / 2$ for massless particles with $\left.s=\left(p_{1}+p_{2}\right)^{2}\right)$ and $d \Phi$ is the usual relativistic phase space. The cross section is well defined despite the virtual gravi-ghost, which gets indirectly defined by what it does. In order to proceed in understanding the ghost, we decompose the scattering amplitude $\mathscr{A}$ as the amplitude $\mathscr{S}_{\mu \nu}$ for the 'scattering' times the gravi-ghost propagator $P_{\mu \nu \alpha \beta}$ of Eq. (3), times the amplitude $\mathscr{D}_{\alpha \beta}$ for the 'decay':

$\mathscr{A}=\mathscr{S}_{\mu \nu} P_{\mu \nu \alpha \beta}\left(k^{2}\right) \mathscr{D}_{\alpha \beta}$.

We show only the gravi-ghost indices, leaving implicit the matter indices. We also leave implicit the usual sum (average) over their initial-state (final-state) components: polarizations, other multiplicities, etc. Inserting $1=\int d^{4} k \delta(k-$ $\left.\sum q_{i}^{\prime}\right)$ times $1=\int d s_{g} \delta\left(k^{2}-s_{g}\right)$ the phase space decomposes as

$d \Phi=d \Phi_{\text {scattering }} \frac{d s_{g}}{2 \pi} d \Phi_{\text {decay }}$,

where $d \Phi_{\text {decay }}$ is the phase space for the decay of a particle with squared mass $s_{g}=k^{2}$ into particles with quadrimomenta $q_{i}^{\prime}$, and $d \Phi_{\text {scattering }}$ is the phase space for producing the gravi-ghost and the final-state particles with momenta $q_{i}$. If the set of final-state particles with momenta $q_{i}$ is empty, $d \Phi_{\text {scattering }}$ contains a Dirac $\delta$ which removes the $d s_{g}$ integral.

In general, the different spin components of intermediate particles give different angular distributions of final-state particles, but have the same decay width, because of Lorentz invariance. Thereby the squared amplitude simplifies after integrating over $d \Phi_{\text {decay }}$. Since the integrated squared decay amplitude only depends on $k$, and since $\mathscr{D}_{\alpha \beta} k_{\alpha}=0$, it has the form

$$
\int d \Phi_{\text {decay }} \mathscr{D}_{\alpha \beta} \mathscr{D}_{\alpha^{\prime} \beta^{\prime}}^{*}=\int d \Phi_{\text {decay }} \sum_{j=\{0,2\}}\left|D^{(j)}\right|^{2} \Pi_{\alpha \beta \alpha^{\prime} \beta^{\prime}}^{(j)}
$$

where

$$
\begin{aligned}
\left|D^{(2)}\right|^{2} & =\frac{1}{5} \Pi_{\alpha \beta \alpha^{\prime} \beta^{\prime}}^{(2)} \mathscr{D}_{\alpha \beta} \mathscr{D}_{\alpha^{\prime} \beta^{\prime}}^{*}, \\
\left|D^{(0)}\right|^{2} & =\Pi_{\alpha \beta \alpha^{\prime} \beta^{\prime}}^{(0)} \mathscr{D}_{\alpha \beta} \mathscr{D}_{\alpha^{\prime} \beta^{\prime}}^{*} .
\end{aligned}
$$


Since $\Pi^{(j)}$ are orthogonal projectors the total squared amplitude simplifies to

$$
\int d \Phi|\mathscr{A}|^{2}=\int d \Phi \sum_{j=\{0,2\}}\left|S^{(j)} D^{(j)} P^{(j)}\right|^{2},
$$

where the squared 'scattering' amplitude summed over gravighost polarizations is

$$
\left|S^{(j)}\right|^{2} \equiv \mathscr{S}_{\mu \nu} \Pi_{\mu \nu \mu^{\prime} \nu^{\prime}}^{(j)} \mathscr{S}_{\mu^{\prime} \nu^{\prime}}^{*}
$$

The cross section splits into its 'scattering' and 'decay' parts as

$$
\begin{aligned}
& \frac{d \sigma}{d \Phi_{\text {scattering }} d s_{g}}=\sum_{j=\{0,2\}} \frac{c_{j} f_{j}^{2}}{4 \pi I}\left|S^{(j)}\right|^{2} \\
& \times\left|P^{(j)}\left(s_{g}\right)\right|^{2} \times \frac{c_{j} f_{j}^{2}}{2} \int d \Phi_{\text {decay }}\left|D^{(j)}\right|^{2} .
\end{aligned}
$$

At $s_{g} \gg M_{j}^{2}$ the modulus squared of the 4-derivative propagator reduces to $1 / s_{g}^{4}$ : the graviton-graviton and the ghostghost terms get cancelled by the ghost-graviton interference term, which vanishes on-shell at $s_{g}=M_{2}^{2}$.

\subsection{Cross section for producing $N$ gravi-ghosts}

The cross section for producing two on-shell gravi-ghosts $g_{\mu_{i} \nu_{i}}$ with momenta $k_{i}$, squared masses $s_{i}=k_{i}^{2}$, spin $j_{i}$, where $i=\{1,2\}$ can be analogously extracted from the onshell part of scattering amplitudes among matter particles mediated by two gravi-ghosts. The phase space decomposes as

$d \Phi=\frac{d s_{1}}{2 \pi} \frac{d s_{2}}{2 \pi} d \Phi_{\text {scattering }} d \Phi_{\text {decay } 1} d \Phi_{\text {decay } 2 .}$.

Proceeding analogously to the previous section, we define as $\mathscr{S}_{\mu_{1} v_{1} \mu_{2} v_{2}}$ the 'scattering' sub-amplitude, such that the cross section for production of two on-shell gravi-ghosts is ${ }^{1}$

$\sigma_{j_{1} j_{2}}=\frac{1}{4 I} \frac{c_{j_{1}} f_{j_{1}}^{2}}{M_{j_{1}}^{2}} \frac{c_{j_{2}} f_{j_{2}}^{2}}{M_{j_{2}}^{2}} \int d \Phi_{\text {scattering }}\left|S^{\left(j_{1}, j_{2}\right)}\right|^{2}$,

where

$$
\left|S^{\left(j_{1}, j_{2}\right)}\right|^{2}=\mathscr{S}_{\mu_{1} v_{1} \mu_{2} v_{2}} \Pi_{\mu_{1} v_{1} \mu_{1}^{\prime} v_{1}^{\prime}}^{\left(j_{1}\right)} \Pi_{\mu_{2} v_{2} \mu_{2}^{\prime} v_{2}^{\prime}}^{\left(j_{2}\right)} \mathscr{S}_{\mu_{1}^{\prime} v_{1}^{\prime} \mu_{2}^{\prime} v_{2}^{\prime}} .
$$

Analogous expressions hold for $N$ gravi-ghosts.

\footnotetext{
${ }^{1}$ Note the absence of a symmetry factor for identical intermediate gravitons $\sigma_{j_{1} j_{2}}$. The appropriate symmetry factor should of course be included in the total cross section $\sigma(\cdots \rightarrow(g \rightarrow \cdots)(g \rightarrow \cdots))$ in case the gravi-ghosts decay into identical particles.
}

2.4 Processes mediated by one on-shell gravi-ghost

As usual, the cross section is dominated by the phase-space region where the gravi-ghost is on-shell, if this is kinematically allowed. In the narrow-width approximation, around the poles at $s_{g} \simeq M_{j}^{2}$ the gravi-ghost squared propagator approximates as

$\left|P^{(j)}\right|^{2} \simeq \frac{\pi}{M_{j}^{5}\left|\Gamma_{j}\right|} \delta\left(k^{2}-M_{j}^{2}\right)$

Thereby Eq. (13) reduces on-shell to

$\frac{d \sigma}{d \Phi_{\text {scattering }}} \simeq \sum_{j=\{0,2\}} \frac{d \sigma_{j}}{d \Phi_{\text {scattering }}} \frac{\Gamma_{j \rightarrow f}}{\left|\Gamma_{j}\right|}$

where

$\Gamma_{j \rightarrow f}=\frac{c_{j} f_{j}^{2}}{2 M_{j}^{3}} \int d \Phi_{\text {decay }}\left|D^{(j)}\right|^{2}$

is the partial decay width of the component of the gravi-ghost with spin $j$ with total decay width $\Gamma_{j}$, and

$d \sigma_{g_{j}}=\frac{1}{4 I} \frac{c_{j} f_{j}^{2}}{M_{j}^{2}}\left|S^{(j)}\right|^{2}=\frac{1}{4 I} \frac{2 c_{j}}{\bar{M}_{\mathrm{Pl}}^{2}}\left|S^{(j)}\right|^{2}$

is the differential cross section for gravi-ghost single production. The factors $\left(c_{j} f_{j} / M_{j}^{2}\right)^{2}$ have been split symmetrically among scattering and decay, getting the standard normalization of decay widths in Sect. 2.5. Although $\Gamma_{j}$ and $d \sigma_{j}$ are negative when the ghost with $c_{2}<0$ is involved, the cross section among matter particles $d \sigma$ is always positive. The negative width signals microscopic acausality $[10,11]$.

The cross section for producing the massless graviton $g$ is $d \sigma_{g}=\frac{1}{4 I} \frac{2}{\bar{M}_{\mathrm{Pl}}^{2}}\left|S^{(g)}\right|^{2} d \Phi_{\text {scattering }} \stackrel{s \gg M_{0,2}^{2}}{\simeq}-d \sigma_{g_{2}}-d \sigma_{g_{0}}$,

with

$$
\begin{aligned}
& \left|S^{(g)}\right|^{2}=\mathscr{S}_{\mu \nu} \Pi_{\mu \nu \mu^{\prime} \nu^{\prime}}^{(g)}, \mathscr{S}_{\mu^{\prime} \nu^{\prime}}^{*}=-\sum_{j} c_{j}\left|S^{(j)}\right|^{2}, \\
& \Pi_{\mu \nu \mu^{\prime} \nu^{\prime}}^{(g)}=-\sum_{j} c_{j} \Pi_{\mu \nu \mu^{\prime} \nu^{\prime}}^{(j)}
\end{aligned}
$$

as obtained from the squared scattering amplitude $\mathscr{S}_{\mu \nu}$ summed over the polarization sum determined by the 4derivative propagator in the limit $s \ll M_{0,2}^{2}$. 
2.5 The gravi-ghost width from $2 \rightarrow 2$ scattering

A $2 \rightarrow 2$ cross section $A B \rightarrow R \rightarrow A^{\prime} B^{\prime}$ between (for simplicity) massless particles and mediated at tree level in $s$-wave by a generic resonance $R$ with spin $j$ (multiplicity $\left.g_{R}=2 j+1\right)$ and mass $M$ can be written as

$\sigma\left(A B \rightarrow R \rightarrow A^{\prime} B^{\prime}\right)=16 \pi \frac{g_{R}}{g_{A} g_{B}} \frac{\Gamma_{R \rightarrow A B} \Gamma_{R \rightarrow A^{\prime} B^{\prime}}}{\left|k^{2}-M^{2}+i k \Gamma\right|^{2}}$.

Specializing the generic gravi-ghost formula to the $2 \rightarrow 2$ case determines the decay widths, in view of their symmetric appearance in Eq. (23).

Explicit evaluation then gives, in a theory with $N_{S}$ real scalars, $N_{f}$ Weyl fermions, $N_{V}$ vectors (all massless or much lighter than $M_{0,2}$ :

$$
\begin{aligned}
& \Gamma_{2}=-\pi M_{2} \frac{f_{2}^{2}}{(4 \pi)^{2}}\left(\frac{N_{s}}{120}+\frac{N_{f}}{40}+\frac{N_{V}}{10}\right), \\
& \Gamma_{0}=\frac{\pi M_{0} f_{0}^{2}}{24(4 \pi)^{2}} \sum_{S}\left(1+6 \xi_{S}\right)^{2} .
\end{aligned}
$$

Direct computation shows that, at tree level, $\Gamma\left(g_{2} \rightarrow g g\right)=$ $\Gamma\left(g_{2} \rightarrow g g_{0}\right)=0$ (see also $\left.[19,20]\right)$, and that $\Gamma\left(g_{2} \rightarrow g_{0} g_{0}\right)$ equals the decay width into a real scalar with mass $M_{0}$ [see Eq. (32)]. In the Standard Model $N_{s}=4, N_{f}=45, N_{V}=12$ so $\Gamma_{2} \approx-16 \mathrm{eV}\left(M_{2} / 10^{10} \mathrm{GeV}\right)^{3}$. In cosmology ghosts are not in thermal equilibrium at $T \gtrsim M_{2}$.

\subsection{The gravi-ghost width from the imaginary part of its propagator}

We start from the simpler case of a non-tachyonic 4derivative scalar containing a normal scalar with mass $m_{1}$ and a ghost with mass $m_{2}>m_{1}$. The tree-level kinetic term (quadratic part of the action) must be

$\Pi(k)=-\left(k^{2}-m_{1}^{2}\right)\left(k^{2}-m_{2}^{2}\right)$,

where $k$ is the quadri-momentum. Indeed, assuming for simplicity $m_{2} \gg m_{1}$, at low $k^{2} \ll m_{2}^{2}$ this reduces to $\Pi \simeq m_{2}^{2}\left(k^{2}-m_{1}^{2}\right)$, which is the usual kinetic term, up to the overall positive normalization factor $m_{2}^{2}$. This shows that the $k^{4}$ term must have negative sign.

Loop corrections due to interactions with matter generate a positive imaginary part, $\operatorname{Im} \Pi \geq 0$, in view of the optical theorem. Ignoring the $k^{4}$ term in the limit $k^{2} \ll$ $m_{2}^{2}$, this means that a normal scalar has a positive width, $\Pi \simeq m_{2}^{2}\left(k^{2}-m_{1}^{2}+i m_{1} \Gamma_{1}\right)$, as well known. In the opposite $k^{2} \gg m_{1}^{2}$ limit, this means that the ghost has a negative width, $\Pi \simeq-k^{2}\left(k^{2}-m_{2}^{2}+i m_{2} \Gamma_{2}\right)$ with $\Gamma_{2} \leq 0$. This sign, following from general considerations, agrees with explicit resummation of the quantum corrections to a ghost propagator [21].

Coming to the gravi-ghost case, one-loop corrections due to normal matter contribute in a way which respects the symmetries of the Lagrangian: each term gets a correction factor Z

$\sqrt{\operatorname{det} g}\left[Z_{0} \frac{R^{2}}{6 f_{0}^{2}}+Z_{2} \frac{\frac{1}{3} R^{2}-R_{\mu \nu}^{2}}{f_{2}^{2}}-\frac{\bar{M}_{\mathrm{Pl}}^{2}}{2} Z_{M} R\right]$.

Massless matter in the loop give $Z_{M}=1$ and

$Z_{2}=1-f_{2}^{2}\left(\frac{N_{s}}{120}+\frac{N_{f}}{40}+\frac{N_{V}}{10}\right) B_{0}(k, 0,0)$,

$Z_{0}=1+\frac{f_{0}^{2}}{24} \sum_{S}\left(1+6 \xi_{S}\right)^{2} B_{0}(k, 0,0)$,

where

$B_{0}(k, 0,0)=\frac{1}{(4 \pi)^{2}}\left[\frac{1}{\epsilon}+\ln \left(-\frac{\bar{\mu}^{2}}{k^{2}}\right)+2\right]$

is the usual Passarino-Veltman function in $d=4-2 \epsilon$ dimensions. Its $1 / \epsilon$ pole reproduces the RGE for $f_{0,2}$ [2]. Inverting the gravi-ghost kinetic term coming from the action of Eq. (26)

$$
\begin{aligned}
& \left(\frac{k^{4}}{c_{2} f_{2}^{2}} Z_{2}+\frac{\bar{M}_{\mathrm{Pl}}^{2} k^{2}}{4} Z_{M}\right) \Pi_{\mu \nu \rho \sigma}^{(2)} \\
& \quad+\left(\frac{k^{4}}{c_{0} f_{0}^{2}} Z_{0}-\frac{\bar{M}_{\mathrm{Pl}}^{2} k^{2}}{2} Z_{M}\right) \Pi_{\mu \nu \rho \sigma}^{(0)}
\end{aligned}
$$

gives the propagator of Eq. (3) with

$$
P^{(j)}\left(k^{2}\right)=\frac{1}{Z_{j} k^{4}-M_{j}^{2} k^{2} Z_{M}} \text { i.e. } \Gamma_{j}=k \operatorname{Im} Z_{j},
$$

which on-shell reproduces the widths in Eq. (24) inserting the imaginary part $\ln (-1)=i \pi$ in agreement with the optical theorem.

\section{Specific processes}

We now make the previous section more concrete computing specific processes. 


\subsection{Decays}

The gravi-ghost decay squared amplitude into a complex scalar with mass $m_{S}$ is

$$
\begin{aligned}
\left|D^{(2)}\right|^{2} & =\frac{\left(s_{g}-4 m_{S}^{2}\right)^{2}}{120}, \\
\left|D^{(0)}\right|^{2} & =\frac{\left[s_{g}\left(1+6 \xi_{S}\right)^{2}+2 m_{S}^{2}\right]^{2}}{12}
\end{aligned}
$$

such that the decay widths at rest are

$$
\begin{aligned}
\Gamma\left(g_{2} \rightarrow S S^{*}\right) & =-\frac{\pi M_{2} f_{2}^{2}}{60(4 \pi)^{2}} \Phi^{5 / 2}, \\
\Gamma\left(g_{0} \rightarrow S S^{*}\right) & =\frac{\pi M_{0} f_{0}^{2}\left(1+6 \xi_{S}+2 m_{S}^{2} / M_{0}^{2}\right)^{2}}{12(4 \pi)^{2}} \Phi^{1 / 2},
\end{aligned}
$$

where $\Phi=1-4 m_{S}^{2} / M_{2,0}^{2}$. The gravi-ghost decay rates into a Dirac fermion with mass $m_{f}$ is

$$
\begin{aligned}
& \Gamma\left(g_{2} \rightarrow f \bar{f}\right)=-\frac{\pi M_{2} f_{2}^{2}}{20(4 \pi)^{2}}\left(1+\frac{8 m_{f}^{2}}{3 M_{2}^{2}}\right) \Phi^{3 / 2}, \\
& \Gamma\left(g_{0} \rightarrow f \bar{f}\right)=\frac{\pi m_{f}^{2} f_{0}^{2}}{6(4 \pi)^{2} M_{0}} \Phi^{3 / 2},
\end{aligned}
$$

where now $\Phi=1-4 m_{f}^{2} / M_{2,0}^{2}$. The spin-0 component decays at tree level only if conformal invariance is broken by $\xi_{S} \neq-1 / 6$ or by particle masses $m_{S}$ or $m_{f}$ [22].

\section{$3.22 \rightarrow 2$ gravitational scattering among scalars}

To start we consider the gravitational scattering $S S^{*} \rightarrow S^{\prime} S^{\prime *}$ among two different complex massless scalars, such that only $s$-wave graviton exchange contributes:

$$
\begin{aligned}
\frac{d \sigma}{d t}= & \frac{1}{2304 \pi s^{2}} \mid f_{2}^{2}\left(s^{2}+6 t s+6 t^{2}\right) P^{(2)}(s) \\
& -\left.f_{0}^{2} s^{2}\left(1+6 \xi_{S}\right)\left(1+6 \xi_{S^{\prime}}\right) P^{(0)}(s)\right|^{2},
\end{aligned}
$$

where $t \equiv\left(p_{1}-q_{1}^{\prime}\right)^{2}$. The interference between the spin 0 and 2 components cancels in the total cross section, reproducing the general formulæ of Sect. 2.2

$$
\begin{aligned}
\sigma= & \frac{s^{3}}{11520 \pi}\left[f_{2}^{4}\left|P^{(2)}\right|^{2}\right. \\
& \left.+5 f_{0}^{4}\left|P^{(0)}\right|^{2}(1+6 \xi s)^{2}\left(1+6 \xi_{S^{\prime}}\right)^{2}\right] \\
\simeq & \begin{cases}s\left[1+5\left(1+6 \xi_{S}\right)^{2}\left(1+6 \xi_{S^{\prime}}\right)^{2}\right] / 2880 \pi \bar{M}_{\mathrm{Pl}}^{4}, & s \ll M_{0,2}^{2}, \\
80 \pi^{2} \Gamma_{2} \mathrm{BR}_{i} \mathrm{BR}_{f} \delta\left(s-M_{2}^{2}\right) / M_{2}, & s \simeq M_{2}^{2}, \\
80 \pi \mathrm{BR}_{i} \mathrm{BR}_{f} / M_{2}^{2}, & s=M_{2}^{2}, \\
{\left[f_{2}^{4}+5 f_{0}^{4}\left(1+6 \xi_{S}\right)^{2}\left(1+6 \xi_{S^{\prime}}\right)^{2}\right] / 11520 \pi s,} & s \gg M_{0,2}^{2} .\end{cases}
\end{aligned}
$$

For $\sqrt{s} \ll M_{0,2}$ the cross section reduces to the Einstein limit, while for $\sqrt{s} \gg M_{0,2}$ exhibits the softer behaviour typical of renormalizable dimensionless theories. Even if $g_{0}$ and/or $g_{2}$ are light enough to be kinematically accessible at present colliders, the cross sections are Planck-suppressed and thereby negligible except close to the pole. At the peak $\sigma$ saturates the unitarity bound, where $\mathrm{BR}_{i(f)}$ is the branching ratio into the initial (final) state. However, a maximal resonant enhancement needs beams with energy resolution $\Delta$ comparable to the decay widths $\left|\Gamma_{0,2}\right|$, otherwise $\sigma \sim M_{2}^{2} / M_{\mathrm{Pl}}^{2} \Delta^{2}$.

\section{$3.32 \rightarrow 2$ scattering among fermions}

The gravitational cross section for $f \bar{f} \rightarrow f^{\prime} \bar{f}^{\prime}$ where $f$ and $f^{\prime}$ are massless different Dirac fermions is

$$
\frac{d \sigma}{d t}=f_{2}^{4} \frac{\left(s^{4}+10 s^{3} t+42 s^{2} t^{2}+64 s t^{3}+32 t^{4}\right)}{2048 \pi s^{2}}\left|P^{(2)}(s)\right|^{2} .
$$

The total cross-section reads

$\sigma=\frac{f_{2}^{4} s^{3}}{5120 \pi}\left|P^{(2)}(s)\right|^{2}$

Colliders use charged fermions, such that the gauge/gravity interference is more important than the above purely gravitational term.

\section{$3.42 \rightarrow 3$ gravitational scattering among scalars}

In the above $2 \rightarrow 2$ scatterings the quadri-momentum $k$ of the gravi-ghost was fixed by kinematics, $p_{1}+p_{2}=k=q_{1}^{\prime}+q_{2}^{\prime}$. We now consider processes where $k$ is free, allowing to better probe the gravi-ghost behaviour. We compute the scattering

$S\left(p_{1}\right)+S^{*}\left(p_{2}\right) \rightarrow \gamma\left(q_{1}\right)+\left[g(k) \rightarrow S^{\prime}\left(q_{1}^{\prime}\right)+S^{\prime *}\left(q_{2}^{\prime}\right)\right]$

which involves one massless vector $\gamma$ coupled to a massless initial-state scalar $S$ with charge $e$, and a gravi-ghost that transforms into neutral massless scalars $S^{\prime} S^{\prime *}$. The $S S^{*} \rightarrow$ $\gamma g$ scattering amplitudes are

$$
\begin{aligned}
& \left|S^{(2)}\right|^{2}=e^{2}\left(\frac{t u}{s}+s_{g}+\frac{s_{g}^{2} s}{6 t u}\right), \\
& \left|S^{(0)}\right|^{2}=e^{2} \frac{s s_{g}^{2}}{3 t u}\left(1+6 \xi_{S}\right)^{2} .
\end{aligned}
$$

The decay amplitudes are given in Eq. (31). In addition to the usual logarithmic IR enhancement, there is a new kind of IR enhancement in the spin- 2 sector that arises when the gravi-ghost has small $k^{2}=s_{g} \ll s$ (while the individual components of its quadri-momentum $k_{\mu}$ can be large), which 
arises because the intermediate gravi-ghost squared propagator has 4 powers of momentum. Assuming, for simplicity, a scattering squared energy $s$ so high that terms suppressed by powers of $s_{g} / s$ can be neglected, the cross section mediated by the spin- 2 gravi-ghost is

$$
\begin{gathered}
\sigma\left(S S^{*} \rightarrow \gamma S^{\prime} S^{\prime *}\right) \simeq \int_{0}^{s} \frac{d s_{g}}{\pi}\left[\frac{f_{2}^{2} e^{2}}{8 \pi}\right]\left|P^{(2)}\left(s_{g}\right)\right|^{2}\left[\frac{f_{2}^{2}}{8 \pi} \frac{s_{g}^{2}}{120}\right] \\
\simeq \frac{e^{2} f_{2}^{4}}{46080 \pi^{3}} \int_{0}^{s} \frac{d s_{g}}{\left|s_{g}-M_{2}^{2}+i M_{2} \Gamma_{2}\right|^{2}} .
\end{gathered}
$$

The new IR divergence is qualitatively similar to the usual soft/collinear divergences ${ }^{2}$ with quantitative differences:

(i) a $s_{g}^{2}$ suppression from the decay interaction, which contains two powers of momentum, as typical of spin-2 interactions;

(ii) a higher enhancement from the 4-derivative propagator.

As a result, the new IR enhancement is power-like rather than logarithmic. In the massless limit of agravity this gives a IR divergence, as expected given that in this limit the gravitational potential grows with the distance. In the massive theory, the ghost mass $M_{2}$ cuts the divergence such that the gravi-ghost-mediated $2 \rightarrow 3$ cross section is saturated by the ghost peak $s_{g} \approx M_{2}^{2}$. Thereby the IR enhancement can be described by the cross section for on-shell gravi-ghost production in the $S S^{*} \rightarrow \gamma g_{2}$ sub-process:

$$
\begin{aligned}
\sigma\left(S S^{*} \rightarrow \gamma g_{2}\right) & =-\frac{f_{2}^{2}}{s M_{2}^{2}} \int d \Phi_{\text {scattering }}\left|S^{(2)}\right|^{2} M_{2}^{2} \ll s \\
& \simeq-\frac{e^{2} f_{2}^{2}}{48 \pi M_{2}^{2}}=-\frac{e^{2}}{24 \pi \bar{M}_{\mathrm{Pl}}^{2}} .
\end{aligned}
$$

This negative cross section for ghost production, combined with the negative ghost decay width, yields positive cross sections among matter particles. The cross section for producing the massless graviton equals $\sigma\left(S S^{*} \rightarrow \gamma g\right)=e^{2} / 24 \pi \bar{M}_{\mathrm{Pl}}^{2}$. Above the peak at $s_{g}=M_{2}^{2}$ the two processes interfere negatively, reducing the differential cross section $d \sigma / d s_{g}$.

\section{$3.52 \rightarrow 3$ gravitational scattering among fermions}

We consider $e \bar{e} \rightarrow \gamma[g \rightarrow v \bar{v}]$, which is the fermionic analogue of the scalar process computed in Sect. 3.4: scattering of two charged fermions (electron-positron) into a vector (photon) and a gravi-ghost, that decays into two neutral fermions (neutrinos). The $2 \rightarrow 2$ purely gravitational scat-

\footnotetext{
$\overline{2}$ Which are also present since we assumed massless scalars, and affect the terms subleading in $s_{g} \ll s$.
}

tering sub-amplitudes are, in the limit $m_{e}=0$ :

$$
\begin{gathered}
\left|S^{(2)}\right|^{2}=e^{2}\left[\frac{s_{g}\left(s_{g}^{2}+s^{2}\right)}{16 t u}+\frac{2\left(s_{g}^{2}+s^{2}\right)-s s_{g}-4 t u}{8 s}\right], \\
\left|S^{(0)}\right|^{2}=0 .
\end{gathered}
$$

The cross section mediated by the spin- 2 component in the limit $s_{g} \ll s$ is

$\sigma(e \bar{e} \rightarrow \gamma \nu \bar{\nu})=\int_{0}^{s} \frac{d s_{g}}{\pi}\left[\frac{f_{2}^{2} e^{2}}{48 \pi}\right] \times\left|P^{(2)}\left(s_{g}\right)\right|^{2} \times\left[\frac{f_{2}^{2} s_{g}^{2}}{320 \pi}\right]$,

which has a structure analogous to the scalar case in Eq. (40). The cross section for on-shell ghost production is

$\sigma\left(e \bar{e} \rightarrow \gamma g_{2}\right) \stackrel{s \gg M_{2}^{2}}{\simeq}-\frac{e^{2} f_{2}^{2}}{48 \pi M_{2}^{2}}$

again equal (up to a sign) to the cross section for graviton production.

\subsection{Production of two gravi-ghosts: $S S^{*} \rightarrow g_{j_{1}} g_{j_{2}}$}

In the limit $s,|t| \gg M_{2}^{2}$ and $\xi_{S}=-1 / 6$ we find

$\left|S^{(2,2)}\right|^{2} \simeq \frac{t^{2}(s+t)^{2}}{8 s^{2}}$.

The total $S S^{*} \rightarrow g_{2} g_{2}$ cross section is doubly IR enhanced, and grows with $s$ :

$\sigma_{g_{2} g_{2}} \stackrel{s \gg g_{g}}{\simeq} \frac{f_{2}^{4} s}{960 \pi M_{2}^{4}}=\frac{s}{240 \pi \bar{M}_{\mathrm{Pl}}^{4}}$.

In the same limit $\sigma_{g_{2} g_{2}} \simeq \sigma_{g g} \simeq-\sigma_{g g_{2}} \simeq-\sigma_{g_{2} g}$. The cross section for the production for the scalar components of the graviton $g_{0}$ behaves as the cross section for production of a scalar [22]: $\sigma_{g_{0} g_{2}}=0$ and

$\left|S^{(0,0)}\right|^{2} \simeq M_{2}^{4} \frac{\left(s^{2}+6 s t+6 t^{2}\right)^{2}}{144 s^{4}}, \quad \sigma_{g_{0} g_{0}} \simeq \frac{f_{2}^{4}}{11520 \pi s}$.

\section{Ghostrahlung}

IR and collinear enhancements and divergences are a well studied topic in QED, QCD and gravity. Based on previous experience, we explore the implications of the new IR enhancements present in 4-derivative theories. In Sect. 4.1 we discuss the consequences of soft theorems. In Sect. 4.2 we try to go beyond the soft limit. In Sect. 4.3 we consider 
Tree

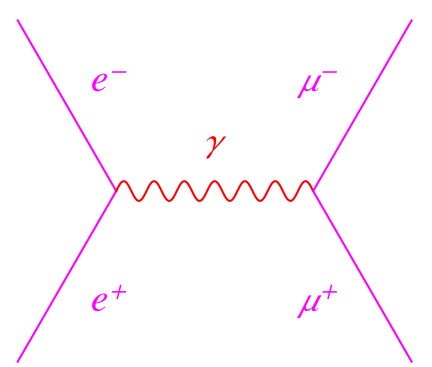

Real

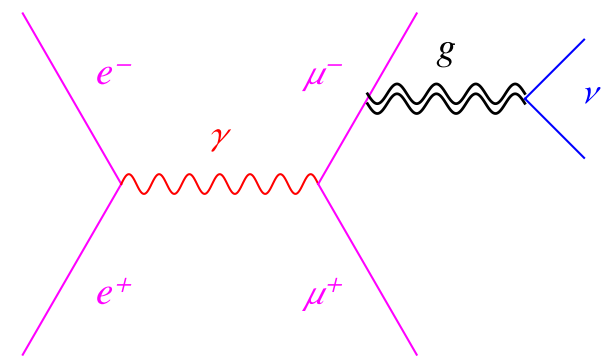

Virtual

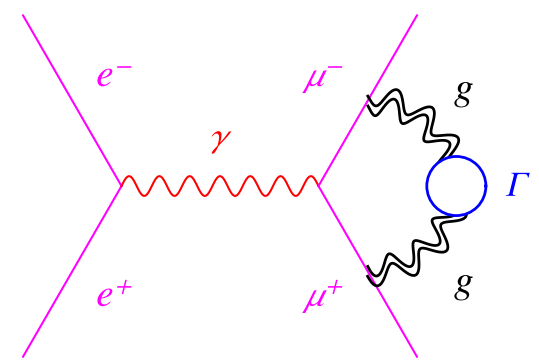

Fig. 3 A sample process. The second diagram, computed with real (Minkowskian) moments, has an IR power enhancement at large gravi-ghost momenta, $k=(E, E, 0,0)$

the approach by Kulish and Faddeev. Based on previous discussions, in Sect. 4.4 we draw our conclusions.

To make the discussion more concrete we compute a toy process $S \rightarrow f \bar{f}$ : a heavy scalar $S$ at rest with mass $m_{S} \gg$ $M_{2}$ decays into massless fermions. Adding one gravi-ghost with energy $E$ to the final state, its energy distribution in terms of $x=2 E / m_{S}$ is

$$
\begin{aligned}
\frac{d N_{g}}{d x} & =\frac{2 f_{2}^{2} m_{S}^{2}}{3(4 \pi)^{2} M_{2}^{2}} \frac{1-7 x / 4+9 x^{2} / 8-3 x^{3} / 8}{x} \\
\frac{d N_{g_{2}}}{d x} & =-\frac{d N_{g}}{d x} \sqrt{1-\frac{4 M_{2}^{2}}{x m_{S}^{2}}}
\end{aligned}
$$

for the graviton and ghost components, respectively. We only wrote the contribution enhanced by $m_{S}^{2} / M_{2}^{2}$, which is the new IR enhancement. The usual IR enhancement produced by the fermion propagator gives the $1 / x$ factor.

\subsection{Soft theorems}

At the diagrammatic level, soft theorems capture IR enhancements in terms of the behaviour of couplings and propagators. In the usual 2-derivative case, emission of spin 1 photons and gluons is enhanced by soft and collinear logarithmic divergences, while emission of spin-2 gravitons is only enhanced by soft logarithmic divergences, because graviton couplings are suppressed as $\theta^{2}$ in the small-angle limit $\theta \ll 1$, cancelled collinear divergences in the propagator [16-18]. The main result of such techniques is that IR divergences cancel between 'real' and 'virtual' corrections when computing appropriate 'IR-safe' observables, which discount too soft particles as unobservable. Indeed, enhanced soft/collinear radiation arises from virtual particles which are almost on shell and propagate for long time, invalidating the difference between real and virtual particles.

\section{IR enhancements in 4-derivative gravity}

IR enhancements are stronger in 4-derivative theories because propagators of massless or light fields with small momentum $k_{\mu}$ are more IR-divergent: $1 / k^{4}$ rather than $1 / k^{2}$. This comes together with the extra issue of understanding what a 'ghost' is, that we addressed by treating the gravi-ghosts as virtual particles in scatterings among matter particles, finding that 4-derivative gravity contains power-like IR enhancements at small $k^{2}$. This includes the region of small $k_{\mu}$ where soft theorems hold, but goes beyond it, including the region where the gravi-ghost splits into collinear ultra-relativistic matter particles.

Adding the Einstein term, the ghost acquires a mass $M_{2}$, and IR divergences get replaced by IR enhancements, saturated around the ghost pole, $k^{2} \simeq M_{2}^{2}$. This is the region where it is more difficult to understand what a ghost is, given that understanding any massive particle needs a nonperturbative resummation of higher-order corrections to their propagator, that acquires Breit-Wigner form.

\section{Soft region}

It is partially useful to explore the implications of soft theorems, which control a part of the new IR enhancement. The amplitude $\mathscr{A}_{\mu \nu}$ for emitting a soft graviton with small momentum $k_{\mu}$ in a process among matter particles with amplitude $\mathscr{A}_{\text {hard }}$ and momenta $p_{i} \gg k$ factorises as [16]

$\mathscr{A}_{\mu \nu}=\mathscr{A}_{\mathrm{hard}} J_{\mu \nu}, \quad J_{\mu \nu}(k)=-\sum_{i} \frac{p_{i \mu} p_{i \nu}}{2 p_{i} \cdot k}$.

All momenta are here in-going, so $p_{i}=-q_{i}$ for final-state particles with out-going momentum $q_{i}$. Summing the squared amplitude over gravi-ghost polarizations gives

$J_{\mu \nu}(k) \Pi_{\mu \nu \mu^{\prime} v^{\prime}}^{(j)}(k) J_{\mu^{\prime} \nu^{\prime}}^{*}(k) \stackrel{k \rightarrow 0}{\simeq}\left|J^{(j)}\right|^{2}$, 
where

$$
\begin{aligned}
\left|J^{(2)}\right|^{2} & \equiv \sum_{i j} \frac{\left(p_{i} \cdot p_{j}\right)^{2}-p_{i}^{2} p_{j}^{2} / 3}{4\left(p_{i} \cdot k\right)\left(p_{j} \cdot k\right)}, \\
\left|J^{(0)}\right|^{2} & \equiv \sum_{i j} \frac{p_{i}^{2} p_{j}^{2} / 3}{4\left(p_{i} \cdot k\right)\left(p_{j} \cdot k\right)}, \\
\left|J^{(g)}\right|^{2} & =-\sum_{j=\{0,2\}} c_{j} J^{(j)}
\end{aligned}
$$

up to terms finite for $k \rightarrow 0$. The cross section among matter particles mediated by a virtual gravi-ghost is given in the soft limit by Eq. (13) with

$$
\left|S^{(j)}\right|^{2} \stackrel{k \rightarrow 0}{\simeq}\left|\mathscr{A}_{\text {hard }} J^{(j)}\right|^{2}
$$

Equation (20) then gives the cross section $\sigma_{g_{j}}$ for producing one on-shell soft gravi-ghost $g_{j}$ in terms of the cross section $\sigma_{\text {hard }}$ without gravi-ghosts:

$d \sigma_{g_{j}} \stackrel{k \rightarrow 0}{\simeq} d \sigma_{\text {hard }} \frac{d^{3} k}{(2 \pi)^{3} 2 E_{j}} \frac{2 c_{j}}{\bar{M}_{\mathrm{Pl}}^{2}}\left|J^{(j)}\right|^{2}$,

where the $J^{(j)}$ factors contain the usual IR enhancement. Applied to our toy process, soft theorems $\left(\left|J^{(2)}\right|^{2}=2 / 3 x^{2}\right.$ adding a soft graviton to a $1 \rightarrow 2$ decay with massless finalstate particles) correctly reproduce the leading $1 / x$ term of Eq. (48) for $x \ll 1 .^{3}$

\section{Cancellation of real with virtual corrections in the soft limit}

We now show that, in the soft limit, the real IR enhancement gets cancelled by virtual corrections. The total amplitude for emission of one soft graviton is given by Eq. (49). Its IR divergence gets cancelled by the virtual correction $\delta \mathscr{A}_{\text {hard }}$, obtained by considering the amplitude for emission of two soft gravi-ghosts, $\mathscr{A}_{\mu \nu \alpha \beta}=\mathscr{A}_{\text {hard }} J_{\mu \nu}(k) J_{\alpha \beta}(-k)$ and connecting them into a one-loop diagram through a gravi-ghost propagator. (The cubic graviton vertex does not contribute to the leading IR divergence). In 4-derivative gravity one gets

$$
\begin{gathered}
\frac{\left.\delta \mathscr{A}\right|_{\text {hard }}}{\mathscr{A}_{\text {hard }}} \stackrel{k \rightarrow 0}{\simeq} \frac{1}{2} \int \frac{d^{4} k}{(2 \pi)^{4}} P_{\mu \nu \alpha \beta} J_{\mu \nu}(k) J_{\alpha \beta}(-k) \\
=-\frac{1}{2} \sum_{j=0,2} \int \frac{d^{4} k}{(2 \pi)^{4}} i c_{j} f_{j}^{2} P^{(j)}(k)\left|J^{(j)}\right|^{2} .
\end{gathered}
$$

\footnotetext{
${ }^{3}$ Even the sub-leading term is captured from the soft formula, if one keeps the $k^{2}$ in the eikonal propagators. For graviton emission from a scattering involving scalars only, this reproduces the full amplitude, because non-soft terms with $k_{\mu}$ at the numerator in the amplitude must be contracted with graviton polarizations, and thereby vanish. Then IR enhancements cancel in the total cross section. Processes involving fermions or vectors contain different contractions with their polarizations, so that the soft limit fails at $x \sim 1$.
}

In order to match it with the real correction of Eq. (53) we perform the integral over $d k_{0}$. Keeping generic signs of the $i \epsilon$ in the two components of the 4-derivative propagator gives

$$
\begin{aligned}
& \int_{-\infty}^{+\infty} \frac{d k_{0}}{2 \pi} \frac{i}{\left[k_{0}^{2}-E^{2} \pm i \epsilon\right]\left[k_{0}^{2}-E^{\prime 2} \pm^{\prime} i \epsilon\right]} \stackrel{\epsilon}{\stackrel{0}{\simeq} \frac{1}{E^{\prime 2}-E^{2}}} \\
& \quad \times\left(\frac{ \pm^{\prime}}{2 E^{\prime}}-\frac{ \pm}{2 E}\right) .
\end{aligned}
$$

The virtual correction decomposes as the sum over the massless graviton $\left(E_{g}=k\right)$, the ghost $\left(E_{2}^{2}=k^{2}+M_{2}^{2}\right)$ and the spin 0 component $\left(E_{0}^{2}=k^{2}+M_{0}^{2}\right)$ :

$$
\begin{gathered}
\frac{\left.\delta \mathscr{A}\right|_{\mathrm{IR}}}{\mathscr{A}} \stackrel{k \rightarrow 0}{\underset{\simeq}{\simeq}}-\frac{1}{2} \int \frac{d^{3} k}{(2 \pi)^{3}} \frac{2}{\bar{M}_{\mathrm{Pl}}^{2}}\left[ \pm \frac{\left|J^{(g)}\right|^{2}}{2 E_{g}}\right. \\
\left. \pm^{\prime} c_{2} \frac{\left|J^{(2)}\right|^{2}}{2 E_{2}} \pm^{\prime} c_{0} \frac{\left|J^{(0)}\right|^{2}}{2 E_{0}}\right] .
\end{gathered}
$$

For the $i \epsilon$ prescription that makes the theory renormalizable, $\pm= \pm^{\prime}=+$, the virtual soft correction cancels the real soft correction of Eq. (53). ${ }^{4}$

The above leading-order cancellation, diagrammatically illustrated in Fig. 3, persists at higher orders, where diagrams with a series of matter bubbles on the gravi-ghost propagator give the dominant IR effect. However, resumming corrections to the gravi-ghost propagator transforms it into a Breit-Wigner with a negative width $\Gamma_{2}<0$, which shifts the ghost pole to the acausal region, behaving as a wrongsign $i \epsilon$ prescription. As a consequence the loop integral of the resummed propagator is not the resummation of the loop integrals: one corresponds to $\epsilon>|\Gamma| \rightarrow 0$, the other to $|\Gamma|>\epsilon \rightarrow 0$.

\subsection{Beyond the soft limit}

The $J$ factors in Eq. (54) give the usual IR enhancements, and the 4-derivative propagator gives the new IR enhancement. Focusing on it, one can ignore the $J$ factors and the external momenta. Then, virtual corrections have the typical form of dimensionless theories, exemplified by dimensionless loop integrals such as $\int d^{4} k / k^{4}$. In the Euclidean they lead to logarithmic divergences, that induce RGE running of the couplings. Power-like IR enhancements appear in the Minkowskian, as its clear from $d^{4} k / k^{4}=d s_{g} / s_{g}^{2} \times d^{3} k / 2 E$ : the integral over $s_{g}=k^{2}$ is power divergent at $s_{g} \rightarrow 0$ (and enhanced in the presence of masses).

Non-soft IR enhancements arise from the extra region where $s_{g}=k^{2}$ is small, while the individual components of

\footnotetext{
4 'Real' and 'virtual' effects can be unified viewing squared amplitudes as imaginary parts of higher loop diagrams. Each higher diagram is separately IR convergent, so that the cancellations mentioned above (at the level of the total amplitude) take part separately between the cuttings of each higher diagram.
} 
$k_{\mu}$ are large. This is a collinear configuration (although different from collinear enhancements in 2-derivative theories), which only exists in the Minkowskian. Collinear divergences do not exist in the Euclidean, where small $k_{E}^{2}>0$ implies that each component of $k_{E}$ is individually small.

IR enhancements beyond the soft limit would get under better control if one could use Euclidean techniques. However ghosts complicate the connection between the Minkowskian and Euclidean theory. One may need an integration contour deformed to lie above the ghost pole, or something along the lines of $[10,11]$ or [12].

\subsection{Asymptotic scattering states}

The physics of IR divergences has been clarified by Kulish and Faddeev [23], with recent developments [24-27]. IR divergences appear when the LSZ formula for cross sections (which assumes that particles are free at asymptotically large distances) is used despite being unapplicable due to the presence of long-range interactions.

2-Derivative theories give Coloumbian-like interactions, which do not induce IR divergences provided that cross sections are computed among scattering states that account for such long-range interactions. The correct scattering states are well known in non-relativistic quantum mechanics. The appropriate relativistic states have been computed in QED [23], and look like a charged particle surrounded by a cloud of soft photons. This approach allows to define a $S$-matrix, not just some IR-safe observables.

The Coulomb force $e^{2} / r^{2}$ vanishes at large distances, giving a soft IR divergence. Agravity in the massless limit produces a constant gravitational force $\sim f_{2}^{2} s$, corresponding to a non-soft IR divergence. Since the force is confining, the only possible scattering states have zero energy [28]. When the Einstein term is included, agravity at large distances $r \gtrsim 1 / M_{0,2}$ predicts to the usual Newton force, which behaves as a Coulomb force with coupling $E / M_{\mathrm{Pl}}$. The associated soft IR divergence changes character when the coupling becomes large, namely in super-Planckian scatterings.

\subsection{Discussion}

Based on the previous discussion, we draw the physical conclusions.

Tree-level $2 \rightarrow 2$ gravity-mediated cross sections are of order $\sigma \sim f_{0,2}^{4} / s$ in the agravity regime $\sqrt{s} \gg M_{0,2}$. They are suppressed by the agravity couplings $f_{0,2}$, that we assume to be small in order to keep the Higgs mass naturally smaller than the Planck mass [2].

However, cross sections get infra-red enhanced by powers of $s / s_{g}$ when more gravi-ghosts are involved, such that a virtual gravi-ghost with momentum $k_{\mu}$ can have $s_{g} \equiv k^{2} \ll$ $s$. The largest cross section is obtained by adding gravi-ghost interactions to a $2 \rightarrow 2$ scattering mediated by order-one matter couplings, like those present in the Standard Model. In such scatterings, the leading-order cross sections for emitting a graviton or a ghost are as large as in Einstein gravity, and violate naive unitarity bounds at $\sqrt{s} \gtrsim M_{\mathrm{Pl}}$.

The total number of radiated gravitons is well approximated by soft limits. Resummation of soft radiation shows that it leaves total cross sections roughly unchanged in view of cancellations between real and virtual effects [16].

The total radiated energy is instead dominated by hard gravitons, with $E \sim \sqrt{s}$.

In Einstein gravity, large cross section arise because of UV divergences: the theory is non-renormalizable and Planckian gravitons are strongly coupled. Thereby, after being emitted, they re-scatter giving rise to complicated higher-order phenomena, in particular formation of black holes which has been conjectured to lead to classicalization (see $[29,30]$ for recent studies).

Agravity is renormalizable and the same tree-level cross section arise because of IR enhancements. The key physical difference with Einstein theory is that (super)Planckian gravitons are weakly coupled: after being radiated they simply carry away their energy. This is why, to focus on the largest most problematic cross section, we gravitationally dressed scatterings mediated by order-one matter couplings, rather than by gravity. The fraction of the total energy that is radiated to gravi-ghosts is $\sim B /(1+B)$, where $B \sim$ $f_{2}^{2} s /\left(4 \pi M_{2}\right)^{2} \sim s /\left(4 \pi M_{\mathrm{Pl}}\right)^{2}$ is the soft emission factor of [16]. This implies that, because most of the energy is lost to gravi-ghost radiation, super-Planckian scatterings get effectively down-graded to Planckian, which have cross sections within unitarity bounds. The new IR enhancement is non-soft: the non-cancellation of real and virtual corrections allows for large corrections to the cross sections.

The super-Planckian energy is radiated away by hard gravi-ghosts which are almost free. Agravity provides a perturbative classicalization mechanism, while non-perturbative black holes play a negligible role around the Planck scale. Indeed the energy content of gravitational fields is smaller in agravity than in Einstein gravity. This can be estimated as the Newton potential evaluated at $r \sim 1 / M_{0,2}$, and can be precisely computed in specific cases: for example the one-loop gravitational correction to the mass $M$ of a Dirac fermion is $\Delta M=5 f_{2}^{2} M^{2} / 96 \pi M_{2}$, indicating that Einstein black holes arise in agravity at $M_{\mathrm{BH}} \gtrsim M_{\mathrm{Pl}} / f_{2}[2,31]$.

\section{Conclusions}

4-Derivative gravity is renormalizable thanks to extra graviton components, especially a spin- 2 ghost with mass $M_{2}$, to be quantised with positive energy. We computed the ghost 
behaviour à la Lee-Wick: by viewing ghosts as virtual particles that mediate scattering among ordinary matter particles.

We restricted our attention to tree-level processes, which teach a significant amount of physics, and which are not affected by details of quantisations proposed to make sense of ghosts at higher orders.

Some cross section have the expected good behaviour typical of renormalizable interactions, being softer than analogous cross sections in Einstein theory. Cross sections for on-shell ghost production can be enhanced colliding beams with energy spread as small as the ghost decay width $\Gamma_{2} \sim-M_{2}^{3} / M_{\mathrm{Pl}}^{2}$. The negative sign signals micro-acausality, which can be probed by an observer at large distance through a $2 \rightarrow 2$ process among matter particles mediated in the $s$ channel by the ghost, measuring that the secondary vertex is displaced in the unusual direction, as if the ghost decayed before being produced.

However, other cross sections where gravi-ghosts have small virtuality do not have the expected behaviour typical of renormalizable interactions. Interpreted in terms of real gravi-ghosts, we find that tree-level cross sections for emitting $n$ gravitons grow as $\left(s / M_{\mathrm{Pl}}^{2}\right)^{n} / s$. These cross sections are as bad as in Einstein gravity, as they are not affected by the extra physics present in agravity. Furthermore, tree-level cross sections for emitting one gravitational ghost behave in the same way.

In Einstein gravity, such non-unitary super-Planckian cross sections are a manifestation of the UV-divergent behaviour typical of non-renormalizable theories.

In agravity, such large cross sections arise because of a new kind of IR enhancement, typical of 4-derivative theories, and due to the small-momentum behaviour of a propagator with 4 powers of momentum. This enhancement is a remnant of IR divergences present in the mass-less limit of agravity, where emission of each gravi-ghost with virtuality $s_{g}=k^{2}$ is accompanied by a factor $\int d s_{g} / s_{g}^{2}$. In Minkowski space, $k^{2}$ can be small even if the components $k_{\mu}$ are large, such that the new IR effect extends beyond the soft region.

In Einstein gravity, super-Planckian scattering is accompanied by large energy losses dominated by hard gravitons with Planckian energy. Gravitational fields contain most of the energy and Planckian gravitons are strongly coupled: they re-scatter forming macroscopic black holes, which possibly lead to classicalization.

In agravity, graviton radiation is accompanied by ghost radiation. More importantly, the energy in gravitational fields is negligible and the Planckian gravi-ghosts are weakly coupled: they merely carry away energy, downgrading scatterings to sub-Planckian and thereby screening super-Planckian physics. We argued that a resummation of initial-state gravighost radiation can significantly affect the cross-sections (analogously to how QED radiation induces the radiative return of the $Z$-peak), bringing them down within unitarity bounds. This conclusion is consistent with the KulishFaddeev understanding of IR divergences as an effect of longrange dynamics, taking into account that 4-derivative gravity gives, in the mass-less limit, a confining gravitational interaction.

Acknowledgements The authors thank Rashmish Mishra and Damiano Anselmi for useful discussions. This work was supported by the ERC grant NEO-NAT and by the grants IUT23-6, PUT799, by EU through the ERDF CoE program grant TK133 and by the Estonian Research Council via the Mobilitas Plus grant MOBTT5.

Open Access This article is distributed under the terms of the Creative Commons Attribution 4.0 International License (http://creativecomm ons.org/licenses/by/4.0/), which permits unrestricted use, distribution, and reproduction in any medium, provided you give appropriate credit to the original author(s) and the source, provide a link to the Creative Commons license, and indicate if changes were made.

Funded by SCOAP ${ }^{3}$.

\section{References}

1. K.S. Stelle, Renormalization of higher derivative quantum gravity. Phys. Rev. D 16, 953 (1976)

2. A. Salvio, A. Strumia, Agravity. JHEP 1406, 080 (2014). arXiv: 1403.4226

3. A. Salvio, A. Strumia, Quantum mechanics of 4-derivative theories. Eur. Phys. J. C 76, 227 (2016). arXiv: 1512.01237

4. P.D. Mannheim, Solution to the ghost problem in fourth order derivative theories. Found. Phys. 37, 532 (2006). arXiv:hep-th/0608154

5. M. Raidal, H. Veermäe, On the quantisation of complex higher derivative theories and avoiding the Ostrogradsky ghost. Nucl. Phys. B 916, 607 (2017). arXiv:1611.03498

6. A. Strumia, Interpretation of quantum mechanics with indefinite norm. arXiv: 1709.04925

7. A. Salvio, Quadratic gravity. Front. Phys. 6, 77 (2018). arXiv: 1804.09944

8. T.D. Lee, G.C. Wick, Negative metric and the unitarity of the $S$ matrix. Nucl. Phys. B 9, 209 (1969)

9. T.D. Lee, G.C. Wick, Finite theory of quantum electrodynamics. Phys. Rev. D 2, 1033 (1970)

10. S. Coleman, Acausality, in Erice 1969, Ettore Majorana School on subnuclear phenomena (New York, 1970) pp. 282-387

11. B. Grinstein, D. O'Connell, M.B. Wise, Causality as an emergent macroscopic phenomenon: the Lee-Wick $O(N)$ model. Phys. Rev. D 79, 105019 (2008). arXiv:0805.2156

12. D. Anselmi, M. Piva, A new formulation of Lee-Wick quantum field theory. JHEP 1706, 066 (2017). arXiv: 1703.04584

13. C.F. von Weizsacker, Radiation emitted in collisions of very fast electrons. Z. Phys. 88, 612 (1934)

14. E.J. Williams, Nature of the high-energy particles of penetrating radiation and status of ionization and radiation formulae. Phys. Rev. 45, 729 (1934)

15. G. Altarelli, G. Parisi, Asymptotic freedom in parton language. Nucl. Phys. B 126, 298 (1977)

16. S. Weinberg, Infrared photons and gravitons. Phys. Rev. 140, B516 (1965)

17. R. Akhoury, R. Saotome, G. Sterman, Collinear and soft divergences in perturbative quantum gravity. Phys. Rev. D 84, 104040 (2011). arXiv:1109.0270 
18. M. Beneke, G. Kirilin, Soft-collinear gravity. JHEP 1209, 066 (2012). arXiv:1207.4926

19. J.F. Donoghue, G. Menezes, Gauge assisted quadratic gravity: a framework for UV complete quantum gravity. Phys. Rev. D 97, 126005 (2018). arXiv: 1804.04980

20. D. Anselmi, M. Piva, Quantum gravity, fakeons and microcausality. arXiv:1806.03605

21. B. Fornal, B. Grinstein, M.B. Wise, Lee-Wick theories at high temperature. Phys. Lett. B 674, 330 (2009). arXiv:0902.1585

22. A. Salvio, A. Strumia, Agravity up to infinite energy. Eur. Phys. J. C 78, 124 (2018). arXiv:1705.03896

23. P.P. Kulish, L.D. Faddeev, Asymptotic conditions and infrared divergences in quantum electrodynamics. Teoreticheskaya i Mathematicheskaya Fizika 4(2), 170 (1970)

24. J. Ware, R. Saotome, R. Akhoury, Construction of an asymptotic S matrix for perturbative quantum gravity. JHEP 1310, 159 (2013). arXiv: 1308.6285

25. C. Gomez, M. Panchenko, Asymptotic dynamics, large gauge transformations and infrared symmetries. arXiv:1608.05630
26. D. Kapec, M. Perry, A.-M. Raclariu, A. Strominger, Infrared divergences in QED, revisited. Phys. Rev. D 96, 085002 (2017). arXiv: 1705.04311

27. D. Carney, L. Chaurette, D. Neuenfeld, G. Semenoff, On the need for soft dressing. JHEP 1809, 121 (2018). arXiv: 1803.02370

28. D.G. Boulware, G.T. Horowitz, A. Strominger, Zero energy theorem for scale invariant gravity. Phys. Rev. Lett. 50, 1726 (1983)

29. G. Dvali, C. Gomez, R.S. Isermann, D. Lüst, S. Stieberger, Black hole formation and classicalization in ultra-Planckian $2 \mathrm{~N}$ scattering. Nucl. Phys. B 893, 187 (2015). arXiv:1409.7405

30. M. Ciafaloni, D. Colferai, F. Coradeschi, G. Veneziano, Unified limiting form of graviton radiation at extreme energies. Phys. Rev. D 93, 044052 (2016). arXiv: 1512.00281

31. H. Lu, A. Perkins, C.N. Pope, K.S. Stelle, Black holes in higher-derivative gravity. Phys. Rev. Lett. 114, 171601 (2015). arXiv: 1502.01028 